\title{
Carboxyl-functionalized mesoporous silica-carbon composites as highly efficient adsorbents in liquid phase
}

\author{
Patricia Valle-Vigón, Marta Sevilla and Antonio B. Fuertes* \\ Instituto Nacional del Carbón (CSIC), P. O. Box 73, 33080-Oviedo, Spain \\ ${ }^{*}$ Corresponding author (E-mail: abefu@incar.csic.es)
}

\begin{abstract}
Mesoporous silica-carbon composites functionalized with acidic oxygen groups were prepared by oxidation of a layer of carbon deposited inside the silica pores. The synthesis procedure involved the following steps: a) removal of the silica surfactant, b) impregnation of the silica pores with a carbon precursor, c) carbonization and d) oxidation with ammonium persulfate. The resulting silica-carbon composites contained around 25-30 wt \% of carbonaceous matter with a high density of acid oxygen groups attached to the deposited carbon (i.e. $-\mathrm{COOH},-\mathrm{C}=\mathrm{O}$ and $-\mathrm{OH}$ ). The structural characteristics of the parent silica were retained by the oxidized composite materials, which exhibit a high surface area, a large pore volume and a well-ordered porosity made up of uniform mesopores. The oxygen-functionalized silica-carbon composites were found to be excellent adsorbents of basic dyes (e.g. methylene blue) and heavy metals (i.e. $\mathrm{Cu}^{2+}, \mathrm{Zn}^{2+}$ and $\mathrm{Pb}^{2+}$ ) in aqueous media.
\end{abstract}

Keywords: Mesoporous silica, carbon, composite, oxidation, carboxyl, adsorption. 


\section{Introduction}

Ever since their discovery mesostructured silica materials have generated widespread interest due to their unique textural properties (a well-organized pore arrangement, a well-defined morphology, and large porosity made up of tunable mesopores $[1,2])$. These properties make ordered silica materials attractive for a wide range of applications such as selective adsorption [3], drug delivery [4] or immobilization of biomolecules [5]. Recently, researchers have turned their attention to the functionalization of mesoporous silicas by introducing organic moieties that modify their surface chemical properties [6] Among the organic functionalities attached to the silica surface, acidic oxygen containing groups (i.e., carboxylic, phenolic, and lactonic groups) and especially carboxyl groups seem to be particularly interesting for adsorbing pollutants, immobilizing biomolecules or attaching other ligands [7]. This exceptional adsorption capacity is due to an enhancement of their negative charge density in neutral or basic aqueous media which gives rise to specific binding sites for adsorbates through the deprotonation of oxygenated functionalities. Thus, Li et al. prepared carboxylicfunctionalized mesoporous silica and demonstrated their improved efficiency in removing dyes than the original mesoporous silica [8]. Likewise, Garrone et al. tested the retention properties of carboxy-containing mesoporous silica for removing heavy metal ions [9]. However, the preparation of these functionalized materials entails expensive and complex procedures, generally based on the hydrolysis of cyanidemodified silica with sulphuric acid, which severely limits their applicability [10]. In contrast carbon materials are easily functionalized with oxygen groups and the incorporation of a thin carbon layer coating the pores of mesostructured silica could provide a simple and easily applicable way to introduce carboxylic acid groups into mesoporous materials. The resulting silica-carbon composite would then combine an 
easy functionalizability with the structural properties of mesostructured silica materials. Several synthesis strategies for the deposition of a carbon layer inside the silica network have been reported, such as the chemical vapour deposition process (CVD) [11] or the use of surfactants as carbon source [12]. Recently, we presented a novel route for the fabrication of silica-carbon composites by introducing a homogeneous layer of carbon to cover the silica pores [13]. To ensure a uniform carbon covering, a high concentration of silanol groups was retained on the silica support by using a mild surfactant removal procedure.

Oxidation processes designed to introduce oxygen-containing groups into carbon material have been commonly applied to microporous activated carbons but only a few reports can be found for mesoporous carbon materials [14]. For instance, Vinu et al. applied an oxidation process to templated mesoporous carbons and demonstrated that oxidized materials are more suitable for the manipulation of bulky biomolecules than non-oxidized materials [15]. Surface oxygen functionalities can be used as anchoring points for other functionalities such as amine, thiol or acyl chloride groups using grafting procedures to attach nanoparticles with catalytic properties, for introducing large biomolecules useful in drug delivery, or for tailoring the pore size of the materials [16]. In this study, we present for the first time a procedure for synthesizing mesostructured silica-carbon composites with a carbon layer highly functionalized with acidic oxygenated groups. For this purpose we selected ammonium persulfate (APS) as oxidizing agent due its capacity to generate a high density of surface acidic carboxylic functionalities [17]. Furthermore, we analyzed the behaviour of these oxidized composites as sorbents for the adsorption of a basic textile dye (i.e. methylene blue) and the removal of heavy metals (i.e. $\mathrm{Cu}^{2+}, \mathrm{Zn}^{2+}$ and $\mathrm{Pb}^{2+}$ ions) in an aqueous medium. 


\section{Experimental Section}

\subsection{Synthesis of oxygen functionalized silica-carbon composites}

SBA-15 and KIT-6 mesostructured silica materials were synthesised according to the procedures reported by Zhao et al. [18] and Kleitz et al. [19] respectively. To remove the surfactant, the as-synthesized silica-surfactant materials were solvent-extracted using $4 \mathrm{~mL}$ of a concentrated $\mathrm{HCl}$ solution and $200 \mathrm{~mL}$ of ethanol (95\%) per $1 \mathrm{~g}$ of the as-prepared sample. This procedure was performed twice at $80^{\circ} \mathrm{C}$, and after each extraction, the silica was filtered, washed with ethanol and water and then dried.

The preparation of the silica-carbon composites was carried out according to the procedure reported by Nishihara et al. using 2,3-dihydroxynaphthalene (DN) as carbon precursor [20]. $1.5 \mathrm{~g}$ of silica was added to a solution of $3 \mathrm{~g}$ of $\mathrm{DN}$ in $75 \mathrm{~mL}$ of acetone and the mixture was stirred for several hours at room temperature in a closed vessel. The acetone was then left to evaporate at room temperature overnight and the sample was dried at $80^{\circ} \mathrm{C}$. The solid mixture was heat-treated at $300^{\circ} \mathrm{C}$ for $1 \mathrm{~h}$ under a $\mathrm{N}_{2}$ flow. The unreacted DN was removed by washing with acetone and the DN-silica composite was heat-treated under vacuum at temperatures in the $450-550{ }^{\circ} \mathrm{C}$ range for $2 \mathrm{~h}$. The silica-carbon composites thus obtained were denoted as $\mathrm{C} X \mathrm{D}$, where $\mathrm{C}=$ carbon, $X=\mathrm{S}$ or K for SBA-15, KIT-6 silica respectively and D refers to dihydroxynaphthalene.

The carbonaceous layer of the silica-carbon composite was oxidized by means of a saturated solution of $\left(\mathrm{NH}_{4}\right)_{2} \mathrm{~S}_{2} \mathrm{O}_{8}$ in $\mathrm{H}_{2} \mathrm{SO}_{4}(1 \mathrm{M})$ during $60 \mathrm{~h}$ at room temperature and under stirring [21]. The solid product was collected by filtration and washed with abundant distilled water. The oxidized sample was denoted as $C X D$-ox $(X=S$ or $K)$.

\subsection{Characterization of the materials}


Scanning and Transmission Electron images (SEM and TEM) were taken on a Zeiss DSM 942 microscope and on a JEOL (JEM-2000 EX II) microscope operating at 160 $\mathrm{kV}$ respectively. Nitrogen sorption isotherms were performed at $-196^{\circ} \mathrm{C}$ on a Micromeritics ASAP 2020 volumetric adsorption system. The BET surface area was deduced from an analysis of the isotherms in the relative pressure range of 0.04-0.20. The total pore volume was calculated from the amount of nitrogen adsorbed at a relative pressure of 0.99 . The pore size distributions (PSDs) were determined by applying the Kruk-Jaroniec-Sayari (KJS) method to the adsorption branch [22]. Small and wide angle X-ray diffraction (XRD) patterns were obtained on a Siemens D5000 instrument operating at $40 \mathrm{kV}$ and $20 \mathrm{~mA}$, using $\mathrm{CuKa}$ radiation $(\lambda=0.15406 \mathrm{~nm})$. The carbonaceous matter content in the composites was deduced by thermogravimetric analysis on a CI Electronics system. Diffuse reflectance Fourier-Transform Infrared (FT-IR) spectra of the materials were recorded on a Nicolet Magna-IR 560 spectrometer fitted with a diffuse reflection attachment. X-ray photoelectron spectroscopy (XPS) was carried out on a Specs spectrometer, using $\operatorname{MgK} \alpha(1253.6 \mathrm{eV})$ radiation emitted by a double anode at $50 \mathrm{w}$. The binding energies for the high-resolution spectra were calibrated by setting C 1 s to $284.6 \mathrm{eV}$. Temperature programmed desorption (TPD) experiments were performed in a chemisorption analyzer (Micromeritics, Autochem II) equipped with a mass spectrometer (OmniStar 300O). Acid densities were estimated by neutralization titration. The total content of acidic groups (i.e. carboxyl, lactone and phenolic groups) was quantified by titration with $\mathrm{NaOH}(0.05 \mathrm{~N})$, whereas carboxyl groups were estimated by titration with $\mathrm{NaHCO}_{3}(0.05 \mathrm{~N})$ [23]. To determine the pH value of the aqueous suspensions of the samples, $0.1 \mathrm{~g}$ of dry sample was added to $5 \mathrm{~mL}$ of distilled water and the suspension was stirred $24 \mathrm{~h}$ to reach equilibrium. Then, the $\mathrm{pH}$ of suspension was measured [24]. 


\subsection{Dye adsorption and heavy metal removal experiments}

The adsorption experiments of the basic dye, Methylene Blue (MB) (Aldrich) were carried out in closed vessels, at room temperature and using an orbital stirring. In a typical experiment, $5 \mathrm{mg}$ of mesoporous support was dispersed in $20 \mathrm{~mL}$ of dye solution with an initial concentration ranging from 20 to $500 \mathrm{ppm}$ up to a maximum of $24 \mathrm{~h}$. The influence of the $\mathrm{pH}$ was analyzed at neutral to basic values of $\mathrm{pH}$ (7-11) with $\mathrm{KH}_{2} \mathrm{PO}_{4} / \mathrm{Na}_{2} \mathrm{HPO}_{4}$ and Borax/NaOH buffer solutions $25 \mathrm{mM}$. The amount of dye adsorbed into the support was determined by measuring the concentration of $\mathrm{MB}$ in the solution with a UV-vis spectrophotometer (Shimadzu UV-2401PC) at the maximum absorbance of the dye $(\lambda=662 \mathrm{~nm})$.

The capacity of the materials to retain the selected heavy metal ions $\left(\mathrm{Pb}^{2+}, \mathrm{Cu}^{2+}\right.$ and $\mathrm{Zn}^{2+}$ ) was analyzed by using an atomic absorption spectrometer (Shimadzu AA6300). Typically, around $5 \mathrm{mg}$ of support was added to $10 \mathrm{~mL}$ of metal solution in the

$0.25-5 \mathrm{mmol} \cdot \mathrm{L}^{-1}$ concentration range, at an aqueous $\mathrm{pH}$ of 6.5 , in a closed polyethylene bottle and shaken at room temperature during $24 \mathrm{~h}$. The solutions were then separated from the support by means of centrifugation and the adsorption capacities were calculated from the difference between the metal concentrations before and after the retention experiments.

\section{Results and Discussion}

\subsection{Structural properties of the silica-carbon composites}

Carboxyl-functionalized mesoporous silica-carbon composites were prepared following the procedure set out in Scheme 1. In the first step of our synthesis strategy, the asprepared silica is subjected to a mild surfactant removal process designed to ensure the 
preservation of a large number of surface silanol groups. As recently reported, this step is crucial for maximizing the carbon content [13]. As a result, condensation reactions between these silanol groups and the hydroxyl functionalities of the carbon precursor, 2,3-dihydroxynaphtalene, take place. Subsequently, the impregnated sample is subjected to a carbonization process which gives rise to a silica-carbon composite containing around $30 \mathrm{wt} \%$ of carbonaceous matter. Finally, the composite is oxidized with a saturated solution of APS in order to generate a large number of acidic oxygen functional groups attached to the surface of the carbon layer coating the silica pores.

SEM images in Figure S1 (Supporting Information, SI) reveal that, in spite of the large amount of carbon introduced, the external appearance of the composites (i.e. morphology and particle size $\sim 1 \mu \mathrm{m}$ ), does not change in relation to the parent silica, even after the oxidation step (Figure S1c). The carbon deposited causes a reduction of the textural properties (i.e. BET surface area, pore volume and pore size) with respect to the pristine silica, as can be seen from the data listed in Table 1 . The nitrogen sorption isotherms and pore size distributions of SBA-15, KIT-6 and the derived composites are shown in Figure 1. They reveal a well-developed porosity made up of mesopores of a uniform size in the 6 - $8 \mathrm{~nm}$ range. From a comparison of the pore sizes of the silica and composites it can be inferred that the carbon layer has a thickness of $\sim 1.4 \mathrm{~nm}$, which is equivalent to the thickness of four graphene layers. The low angle-range XRD patterns (see Figure S2 in SI) and TEM images (Figure 2) obtained for the silica materials and their corresponding composites before and after the oxidative treatment reveal that the initial structural order is retained after the carbonizationoxidation steps. Dissolution of the silica skeleton of the silica-carbon composites gives rise to templated carbons. Figure S3 (SI) provides the SEM image and the nitrogen sorption isotherm corresponding to the carbon derived from a SBA-15/carbon 
composite. Figure S3b shows a carbon material with large BET surface area $\left(1080 \mathrm{~m}^{2} \cdot \mathrm{g}^{-1}\right)$ and a high porosity made up of uniform mesopores of $~ 3.9 \mathrm{~nm}$. The SEM image in Figure S3a (SI) reveals that the structure of this templated carbon consists of a bundle of nanorods, which indicates that it is a faithful replica of SBA-15 silica pores and suggests that the carbon layer is located exclusively inside the silica pores of the composites. Finally, it is worth noting that, unlike the templated carbons that undergo a substantial loss of structural order after oxidation [15], the silica-carbon composites retain the pore arrangement typical of mesostructured silica even after the oxidative treatment. This is because the silica framework acts as a support which prevents the pore structure from collapsing.

\subsection{Chemical properties of the functionalized composites}

The nature of the surface oxygen functional groups incorporated into the carbon layer was investigated by means of Fourier transform infrared (FT-IR) and X-ray photoelectron (XPS) spectroscopic techniques. Figure 3 shows the FT-IR spectra for the silica-carbon composites before and after functionalization. Three main bands are apparent after the treatment with APS: a) a broad band at $3700-3200 \mathrm{~cm}^{-1}$ due to $-\mathrm{OH}$ stretching vibrations in alcohols or carboxylic acids and b) two peaks at 1750 and 1630 $\mathrm{cm}^{-1}$ ascribed to $\mathrm{C}=\mathrm{O}$ stretching vibrations in lactones or carboxylic acids and quinones or conjugated ketones respectively [21, 25, 26]. Further information about the nature of the oxygenated functionalities was provided by XPS. Figure 4a shows the C 1s corelevel spectra of the silica-carbon sample (CKD) and the corresponding oxidized composite (CKD-ox). The spectra can be deconvoluted into four contributions: carbon in aromatic and aliphatic structures $(\sim 284.7 \mathrm{eV})$; phenol, ether or enol-keto groups (285.8 eV); carbonyl or quinone (287.1 eV); and carboxyl or ester groups (289.1 eV) [27, 28]. Similarly, deconvolution of the O 1 s spectra (Figure 4b) yields the peaks 
corresponding to the oxygen in ketone, lactone or carbonyl (531.2 eV), hydroxyl, phenol, ester, ether, anhydride (533 eV), carboxyl (534.2 eV) and water (536-536.4 eV) groups respectively. Thus, it should be noted that, whereas most of the oxygen present in the silica-carbon composite forms hydroxyl groups with a minor fraction forming carbonyl/quinine and carboxyl groups, after the oxidation step most of the oxygen forms carboxylic groups.

The presence of acid oxygen groups on the surface of the silica-carbon composites, before and after the oxidative treatment, was evaluated by acid-base titrations (Boehm method) and by measuring the $\mathrm{pH}$ values of the aqueous suspension. These measurements reveal a remarkable increase in the density of acid groups in the oxidized composites. Thus, whereas the non-oxidized composites exhibited a low acid density $\left(\sim 0.5 \mathrm{~mol} \cdot \mathrm{g}^{-1}\right)$, oxidation of the silica-carbon composites gave rise to an acidic density of $\sim 1.4 \mathrm{mmol} \cdot \mathrm{g}^{-1}$, with a carboxyl content of $\sim 0.6 \mathrm{mmol} \cdot \mathrm{g}^{-1}$. As a consequence of the incorporation of acid oxygen functional groups, the $\mathrm{pH}$ value of the aqueous suspension changed from 7.3 for the untreated silica-carbon composites to 3.0 for oxidized samples. The surface oxygen groups present in the oxidized sample were also investigated by temperature programmed desorption (TPD) experiments. Figure 5 shows the TPD profiles of $\mathrm{CO}$ and $\mathrm{CO}_{2}$ for the CKD-ox sample. These curves are deconvoluted into several peaks, which can be attributed to different oxygen functional groups [29]. It can be seen that the release of $\mathrm{CO}_{2}$ and $\mathrm{CO}$ up to $600{ }^{\circ} \mathrm{C}$ is due to the decomposition of carboxyl acid groups, whereas the $\mathrm{CO}_{2}$ evolved at higher temperatures corresponds to the decomposition of lactones. Likewise, phenols and other basic groups such as carbonyl and/or quinone-like structures decompose at high temperatures $\left(>800^{\circ} \mathrm{C}\right)$ evolving $\mathrm{CO}[27,30-32]$. The oxygen content associated with the carbon layer, as deduced from the TPD curves, is 1.7 and $1.8 \mathrm{mmol} \cdot \mathrm{g}^{-1}$ for the CKD- 
ox and CSD-ox samples respectively ( 10 wt \% oxygen on a carbon basis). From the results obtained by the TPD experiments it can be deduced that oxidative treatment generates a large number of carboxyl groups $\left(0.5 \mathrm{mmol} \cdot \mathrm{g}^{-1}\right)$, together with carboxyl anhydride $\left(0.6 \mathrm{mmol} \cdot \mathrm{g}^{-1}\right)$, phenol $\left(0.4 \mathrm{mmol} \cdot \mathrm{g}^{-1}\right)$, lactone $\left(0.1 \mathrm{mmol} \cdot \mathrm{g}^{-1}\right)$, and carbonyl/quinone $\left(0.1 \mathrm{mmol} \cdot \mathrm{g}^{-1}\right)$ groups. The peaks $\mathrm{C}_{1}$ and $\mathrm{C}_{2}$ from the $\mathrm{CO}$ curve can be attributed to the decomposition of carboxyl groups. On the other hand, the anhydride groups are probably formed as a consequence of dehydroxylation between adjacent carboxyl groups, whereas lactone functionalities are probably generated by condensation reactions between adjacent carboxyl and phenol groups [21, 31, 32].

\subsection{Dye adsorption and heavy metal removal}

The capacity of oxidized silica-carbon composites to adsorb basic dyes and remove heavy metals from aqueous solutions was also investigated. Figure 6a shows the modification, with time, of the amount of basic dye (methylene blue, MB) adsorbed onto the CSD-ox sample at different $\mathrm{pH}$ values. It is worth noting the fast adsorption rate observed at all the $\mathrm{pH}$ values, with $~ 95 \%$ of the total amount of dye being adsorbed in the first 2 minutes. The highest sorption of MB occurs at $\mathrm{pH} 10$, when the negatively charged acidic groups act as anchoring sites for the positively charged MB molecules. By contrast, at $\mathrm{pH}=7$ a low adsorption uptake is observed as consequence of the weak electrostatic interactions between the partially deprotonated oxygen functionalities and the cationic dye. On the other hand, the slight decrease in the MB adsorption observed at $\mathrm{pH}=11$ could be due to the instability of this dye in stronger basic conditions. Thus, at high $\mathrm{pH}$ values, $\mathrm{MB}$ could react with $\mathrm{OH}^{-}$and be demethylated to other common dyes (Azure A, B and C) [33]. Figure 6b shows the isotherms for the adsorption of methylene blue onto the silica (SBA-15), silica-carbon composite (CSD) and the oxidized samples (CSD-ox) at pH 10. Due to its hydrophobic 
character, the silica-carbon composite (CSD) hardly adsorbs any MB, and adsorbs even less than silica, which is negatively charged in a basic medium. However, after the oxidation step, the CSD-ox composite is able to adsorb an extremely high amount of basic dye, i.e. $\sim 400 \mathrm{mg} \cdot \mathrm{g}^{-1}$, which is among the highest values reported for mesoporous carbon or silica materials. This value is even larger than those obtained for mesoporous silica samples such as MCM-41 (54 $\left.\mathrm{mg} \cdot \mathrm{g}^{-1}\right)$ [34] and SBA-15 (280 $\left.\mathrm{mg} \cdot \mathrm{g}^{-1}\right)$ [35], carboxyl-functionalized mesoporous MCM-41 silica (113 $\mathrm{mg} \cdot \mathrm{g}^{-1}$ ) [34], silica-carbon composites functionalized with sulphonic groups (252 $\left.\mathrm{mg} \cdot \mathrm{g}^{-1}\right)$ [36], mesoporous carbon materials $\left(\sim 260 \mathrm{mg} \cdot \mathrm{g}^{-1}\right)$ [37], and commercial activated carbons $\left(<400 \mathrm{mg} \cdot \mathrm{g}^{-1}\right)$ [38]. The high adsorption of MB dye by CSD-ox may be attributed to the combination of the unique structural characteristics of the synthesized mesoporous silica-carbon composites and the high density of oxygenated acid functionalities, attached to the carbon layer. Clearly, what happens is that, whereas electrostatic interactions between oxygenated groups and the dye molecules resulting in an increase in the adsorption capacity, the large mesopores also ensure a fast diffusion of the dye along the whole pore structure.

The cation-exchange properties of the carboxyl functionalized silica-carbon composites were tested for the removal of $\mathrm{Cu}^{2+}, \mathrm{Pb}^{2+}$ and $\mathrm{Zn}^{2+}$ cations in an aqueous medium. To perform the adsorption experiments we selected a $\mathrm{pH}$ value of $6.5 \pm 0.5$, which is similar to the $\mathrm{pH}$ of normal wastewater treatment. At this $\mathrm{pH}$ value, metal cations are likely to be found as free ions in equilibrium with the solvated species. This is the most appropriate $\mathrm{pH}$ value for adsorption to take place given that higher $\mathrm{pH}$ values tend to precipitate metal species and low $\mathrm{pH}$ values result in a low adsorption because of the increase in competition between the metal ions and protons for adsorption sites. Thus, under these conditions metal species can be expected to be 
electrostatically attracted by the negatively charged acidic surface of the oxidized mesoporous silica-carbon sorbents. Figure 7a shows the adsorption uptakes obtained for KIT-6 silica, the silica-carbon composite (CKD) and the oxidized sample (CKD-ox). As can be seen, very low adsorption uptakes were obtained for silica and the non-oxidized silica-carbon composite. In contrast, large amounts of metal cations were removed by the oxygen-functionalized silica-carbon composite. This confirms the dominant role played by the surface functional groups within the pore structure of the sorbents in removing heavy metals, largely due to electrostatic interactions. Figure $7 \mathrm{~b}$ shows the adsorption isotherm for these heavy metal ions and the CKD-ox sample. The equilibrium data displayed in the figure were fitted to the Langmuir model which allows the maximum adsorption uptake to be calculated. The values thus obtained were 61,82 and $87 \mathrm{mg} \cdot \mathrm{g}^{-1}$ for $\mathrm{Cu}^{2+}, \mathrm{Pb}^{2}, \mathrm{Zn}^{2+}$ respectively (CKD-ox sample). These uptakes are significantly larger than those reported for carboxyl functionalized activated carbon (54 $\mathrm{mg} \mathrm{Cu}^{2+} \cdot \mathrm{g}^{-1}$ ) [39], or silica samples functionalized with amino- (19 $\mathrm{mg} \mathrm{Cu}^{2+} \cdot \mathrm{g}^{-1}$ and $\left.24 \mathrm{mg} \mathrm{Pb}{ }^{2+} \cdot \mathrm{g}^{-1}\right)$ [40] and mercapto- (24 mg Cu${ }^{2+} \cdot \mathrm{g}^{-1}, 26 \mathrm{mg} \mathrm{Pb} \mathrm{Pb}^{2+} \cdot \mathrm{g}^{-1}$ and $11 \mathrm{mg} \mathrm{Zn}^{2+} \cdot \mathrm{g}^{-1}$ ) [41] functional groups, confirming the excellent capacity of these carboxyl functionalized mesoporous silica-carbon composites to serve as sorbents for the removal of heavy metals.

\section{Conclusions}

In summary, we have presented an easy route to synthesize mesoporous silicacarbon materials functionalized with acid oxygen-containing groups. The synthesis procedure consists in the incorporation of a uniform carbon layer such that is covers the silica pores. This carbon layer is then oxidized with a solution of ammonium persulfate. The resulting silica-carbon composites combine the structural properties of the original mesoporous silica materials (i.e. a large BET surface area of $350 \mathrm{~m}^{2} \cdot \mathrm{g}^{-1}$, a large pore 
volume of $\sim 0.5 \mathrm{~cm}^{3} \cdot \mathrm{g}^{-1}$, and a porosity made up of mesopores of $\sim 7 \mathrm{~nm}$ ), with the facile functionalizability by oxidation of the carbon layer deposited within the silica pores. The oxidative treatment of these materials generates a large number of acid oxygen functionalities on the carbon layer, largely in the form of carboxyl groups. Whereas oxidation enhances electrostatic interactions between acid oxygen groups and cationic molecules under basic (i.e. cationic dye) or neutral (i.e. metal cations) conditions, the mesopore structure itself ensures the rapid diffusion of the adsorbate molecules towards the adsorption sites. Thus, the excellent capacity of these materials to act as sorbents of basic dyes (i.e. methylene blue $\sim 400 \mathrm{mg} \cdot \mathrm{g}^{-1}$ ) and heavy metals $\left(\mathrm{Cu}^{2+}: 61 \mathrm{mg} \cdot \mathrm{g}^{-1}, \mathrm{~Pb}^{2+}: 82 \mathrm{mg} \cdot \mathrm{g}^{-1}\right.$ and $\left.\mathrm{Zn}^{2+}: 87 \mathrm{mg} \cdot \mathrm{g}^{-1}\right)$ is confirmed.

Acknowledgments. This work was supported by the Spanish MICINN (Project CQT2011-24776). M.S. and P.V-V. acknowledge Ramon y Cajal and JAE-Predoc contracts respectively. 


\section{$\underline{\text { LEGENDS }}$}

Scheme 1. Illustration of the synthesis route used for preparing mesostructured silica-carbon composites functionalized with oxygenated functional groups.

Figure 1. Nitrogen sorption isotherms (a and b) and pore size distributions (c and d) obtained for SBA-15 and KIT -6 silica, silica-carbon composites and oxidized silicacarbon composites.

Figure 2. TEM images of (a) CSD, (b) CSD-ox, (c) CKD and (d) CKD-ox.

Figure 3. FTIR spectra of the SBA-15/carbon composite and the oxidized sample.

Figure 4. (a) C 1s and (b) O 1s core-level XPS spectra. In order to avoid interferences from the silica framework, the XPS analyses were performed using the corresponding templated carbon derived from samples CKD and CKD-ox.

Figure 5. (a) $\mathrm{CO}$ and (b) $\mathrm{CO}_{2}$ TPD profiles for the CKD-ox sample. Deconvoluted peaks: SA, strongly acidic carboxyl groups; WA, weakly acidic carboxyl groups; CA, carboxyl anhydrides; LD, lactones; PH, phenols; CQ, carbonyl/quinone functionalities.

Figure 6. (a) Modification of the amount of $\mathrm{MB}$ adsorbed with time at different $\mathrm{pH}$ values (CSD-ox sample), (b) adsorption isotherms of MB over the different supports at room temperature and $\mathrm{pH} 10$. The solid lines in (b) represent the best fit of the experimental data to the Langmuir model.

Figure 7. (a) Amount of heavy metal removed by adsorption in the KIT-6 based materials and (b) adsorption isotherms corresponding to the selected metal cations in the CKD-ox sample. The solid lines in $\mathrm{b}$ represent the best fit of the experimental data to the Langmuir model. 
Table 1. Physical properties of the silica, silica-carbon and functionalized silica-carbon composites.

\begin{tabular}{|c|c|c|c|c|c|c|c|c|}
\hline \multirow[b]{2}{*}{ Samples } & \multirow[b]{2}{*}{ Code } & \multirow{2}{*}{$\begin{array}{c}\text { Carbonaceous } \\
\text { matter } \\
(\text { wt \%) })^{\mathrm{a}}\end{array}$} & \multirow{2}{*}{$\begin{array}{c}\text { C content } \\
\text { (wt \%) }\end{array}$} & \multirow{2}{*}{$\begin{array}{c}\text { H content } \\
\text { (wt \%) }\end{array}$} & \multirow{2}{*}{$\begin{array}{l}\text { O content } \\
(\mathrm{wt} \%)^{\mathrm{b}}\end{array}$} & \multicolumn{3}{|c|}{ Textural properties } \\
\hline & & & & & & $\begin{array}{c}\mathrm{S}_{\mathrm{BET}} \\
\left(\mathrm{m}^{2} \cdot \mathrm{g}^{-1}\right)\end{array}$ & $\begin{array}{c}\mathrm{V}_{\mathrm{p}} \\
\left(\mathrm{cm}^{3} \cdot \mathrm{g}^{-1}\right)^{\mathrm{c}}\end{array}$ & $\begin{array}{c}\text { Pore size } \\
(\mathrm{nm})^{\mathrm{d}}\end{array}$ \\
\hline \multirow{2}{*}{ Silica } & SBA-15 & - & - & - & - & 590 & 1.1 & 9.5 \\
\hline & KIT-6 & & - & - & - & 650 & 1.2 & 9.6 \\
\hline \multirow{2}{*}{ Silica-Carbon composites } & CSD & 27.2 & 26.2 & 0.4 & 0.6 & 320 & 0.44 & 6.7 \\
\hline & CKD & 33 & 29.6 & 0.2 & 0.65 & 420 & 0.57 & 7.9 \\
\hline \multirow{2}{*}{ Oxidized samples } & CSD-ox & 25.8 & 22.6 & 0.6 & 2.78 & 310 & 0.43 & 6.5 \\
\hline & CKD-ox & 32.6 & 28.6 & 0.5 & 2.82 & 350 & 0.46 & 7.6 \\
\hline
\end{tabular}


Silica/surfactant hybrid
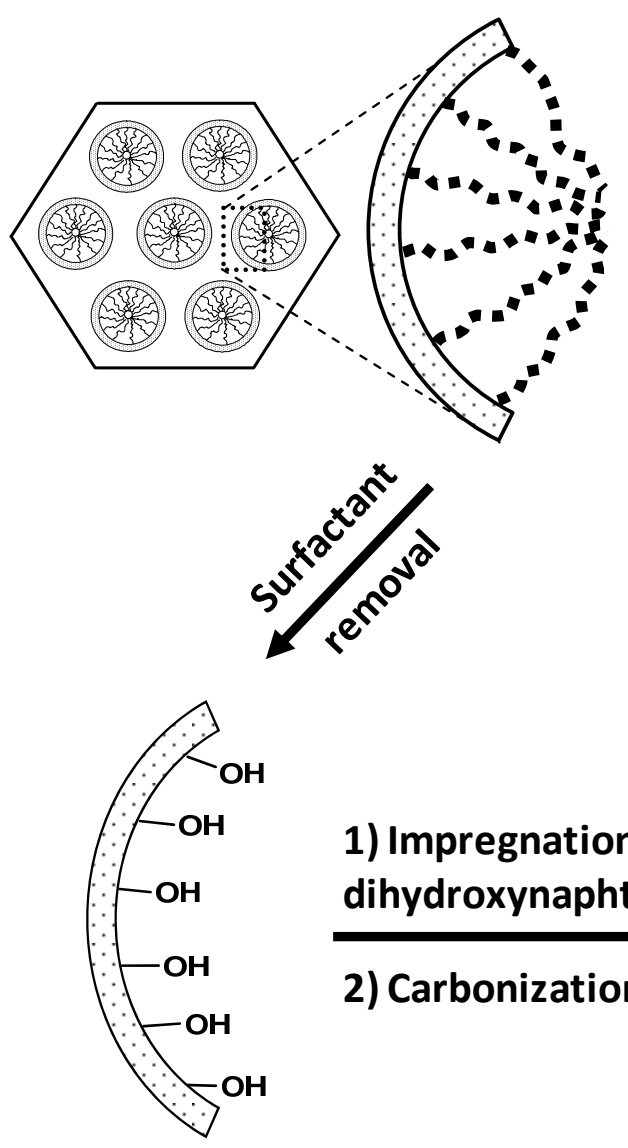

Silica
Oxidized silica-carbon composite
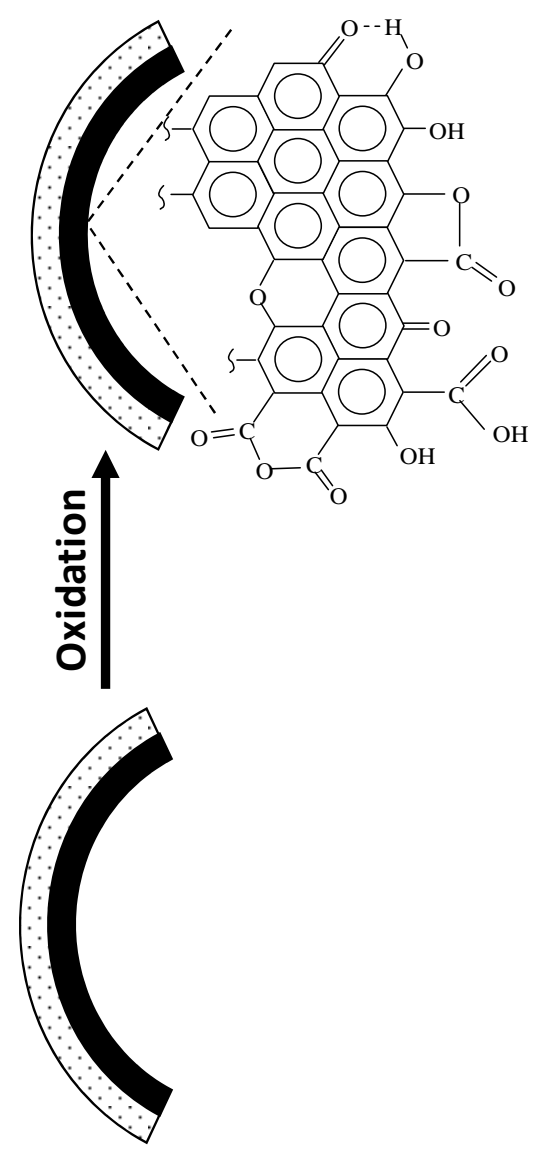

Silica-Carbon

Scheme 1. 
a)

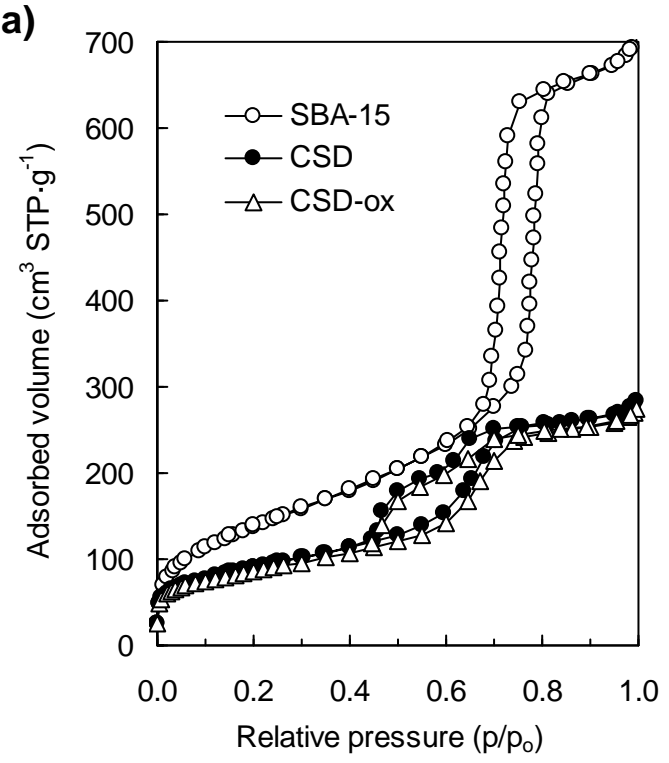

b)

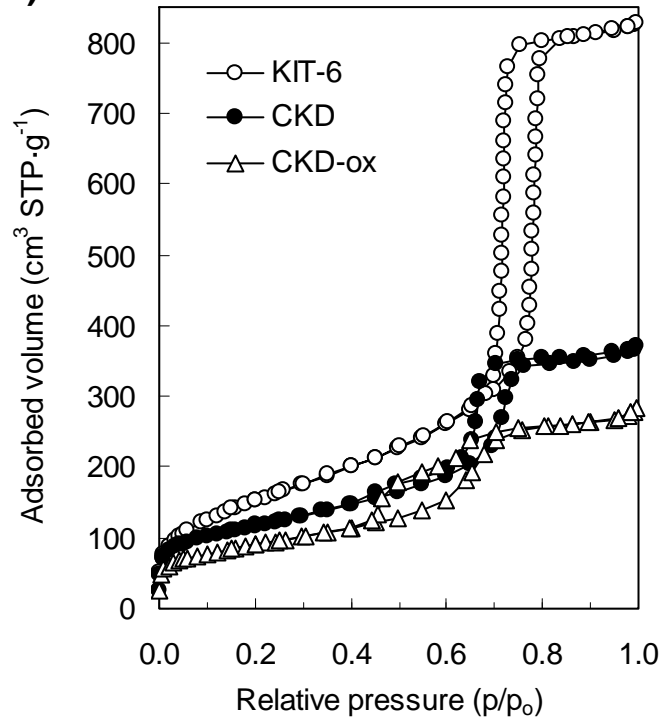

c)

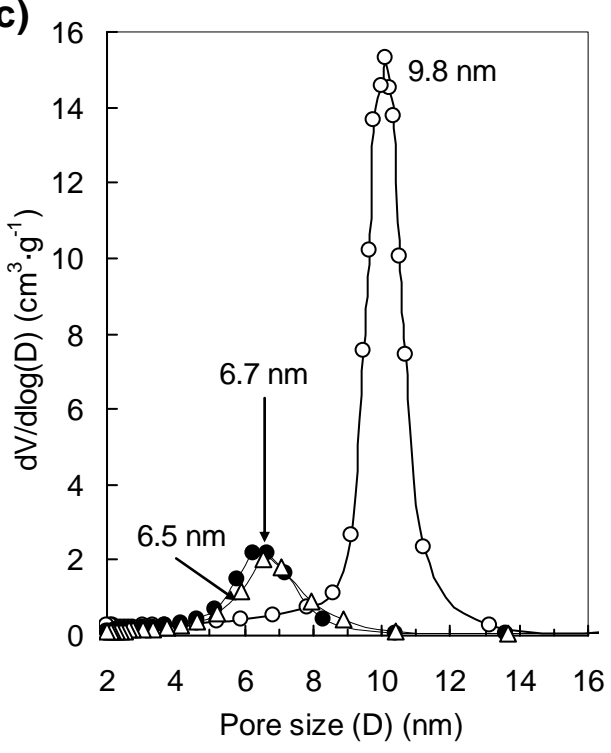

d)

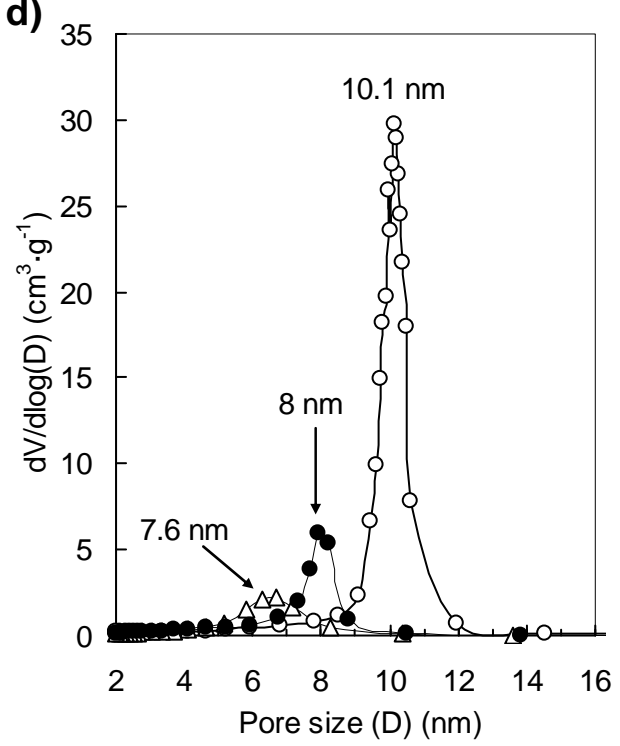

Figure 1. 

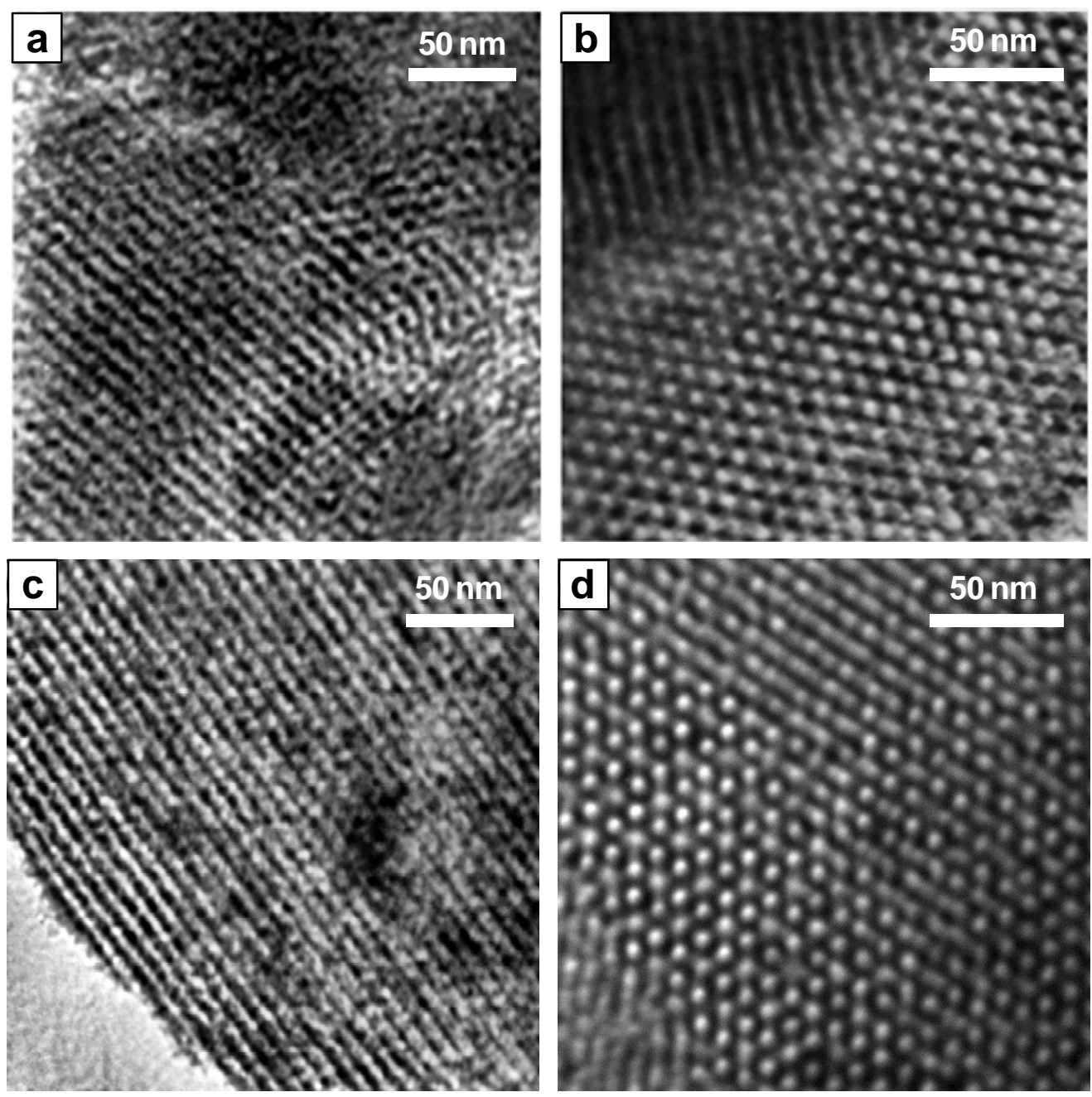

Figure 2. 


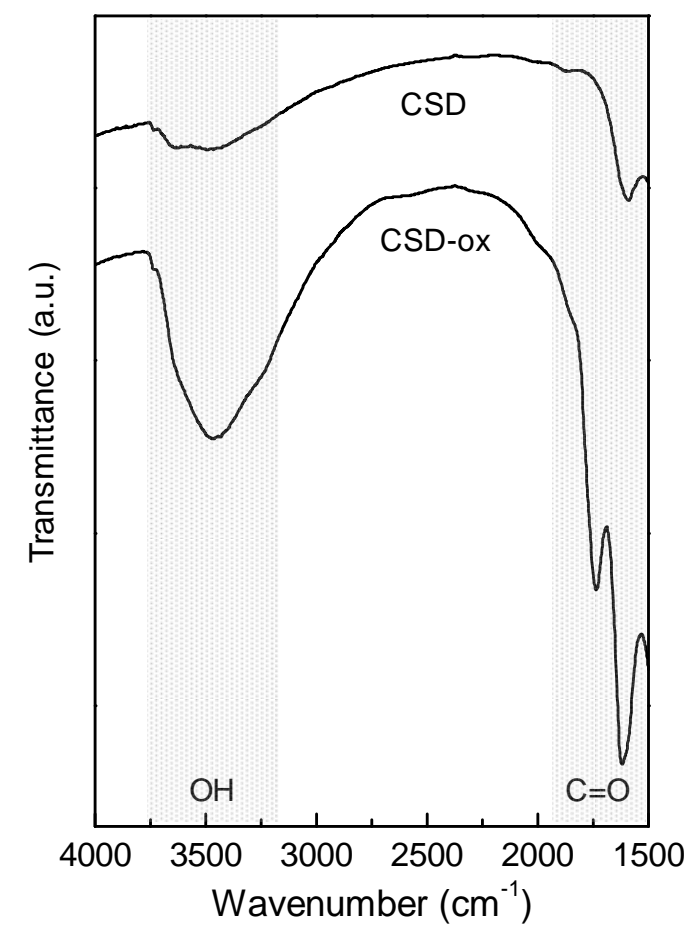

Figure 3.

a)

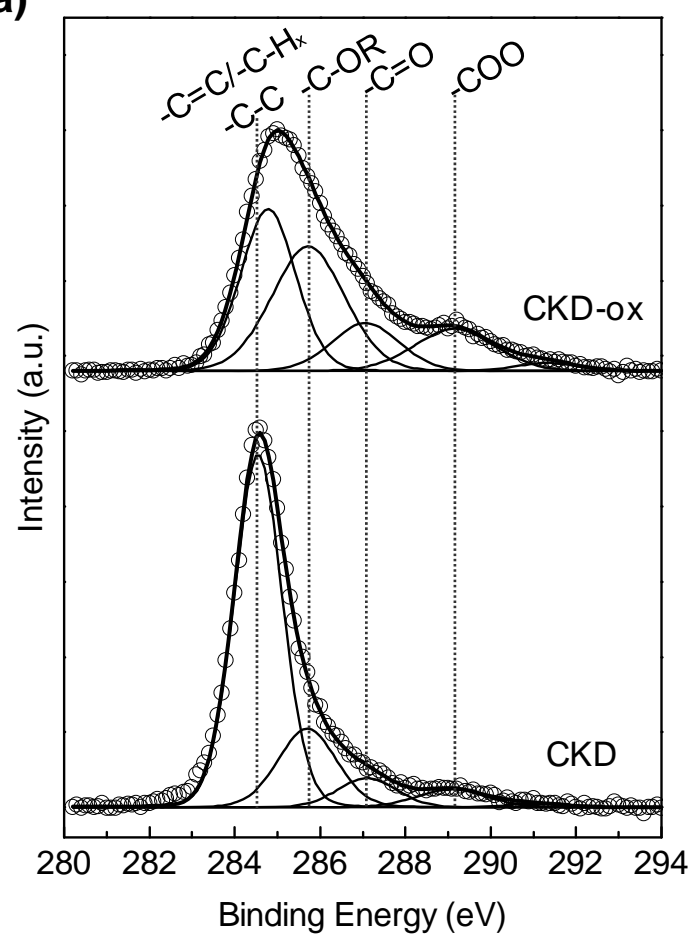

b)

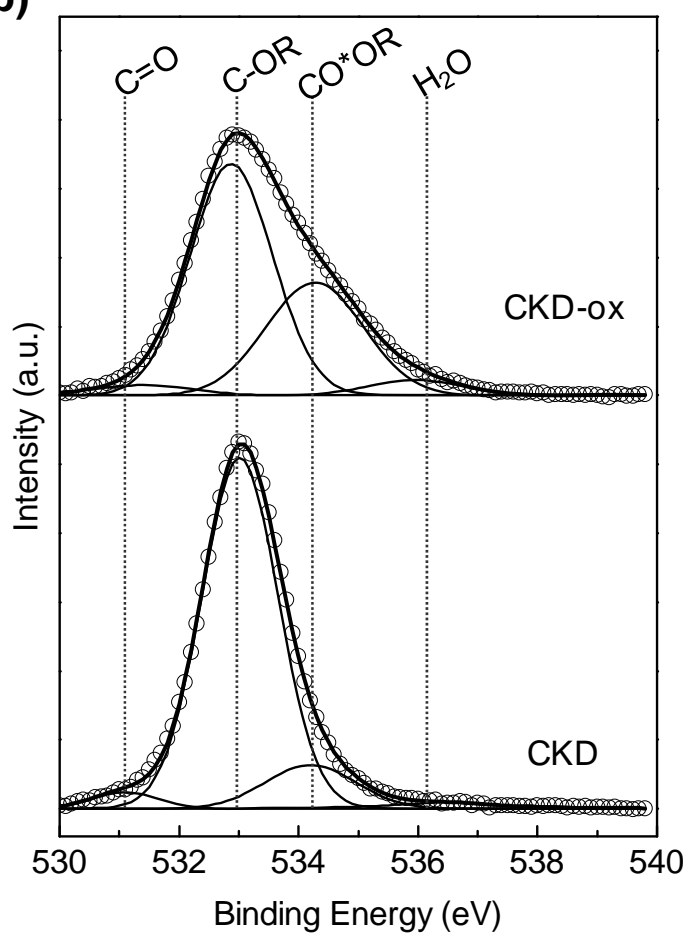

Figure 4. 
a)

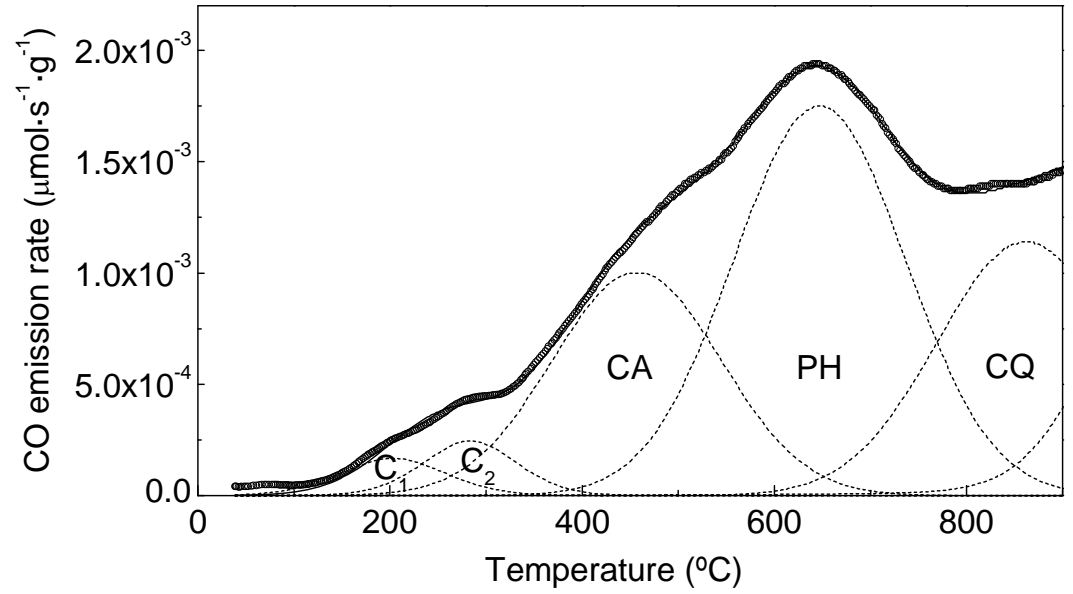

b)

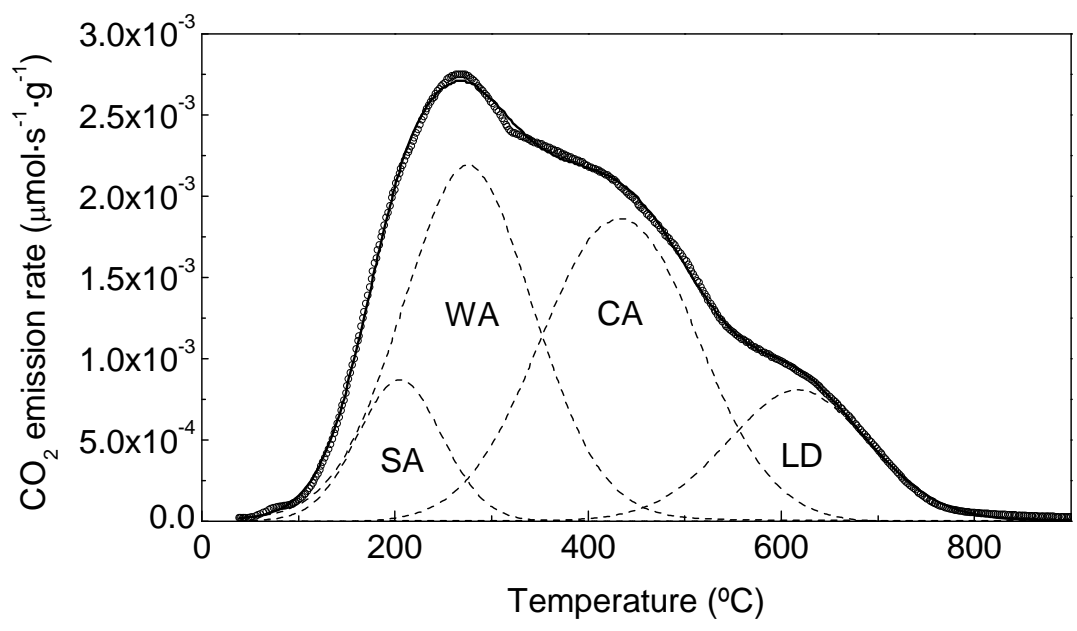

Figure 5. 
a)

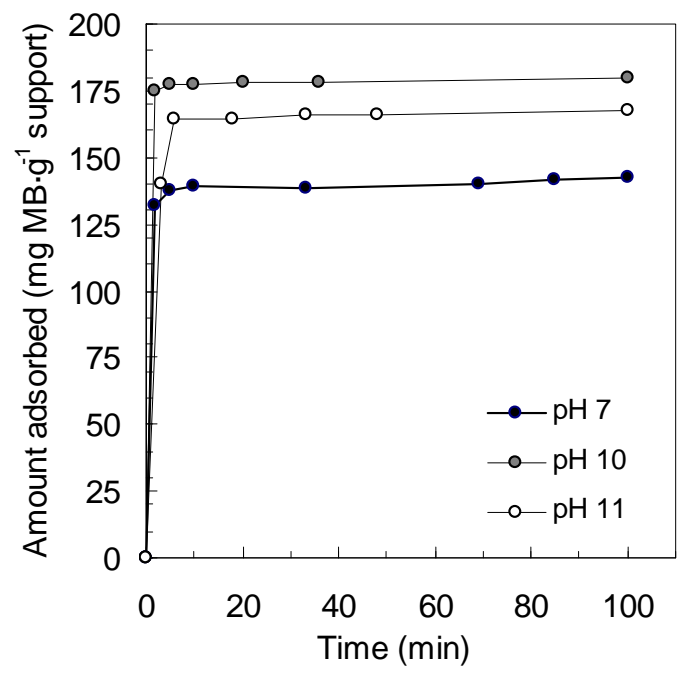

b)

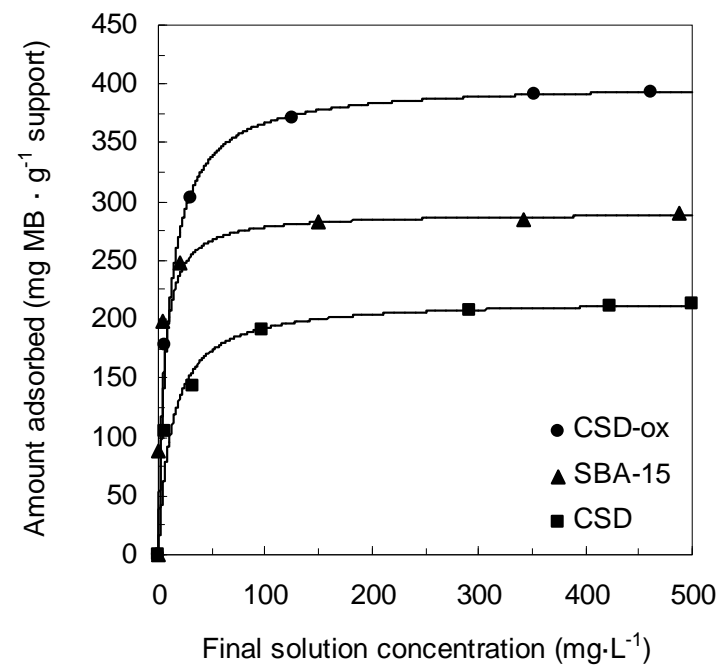

Figure 6.
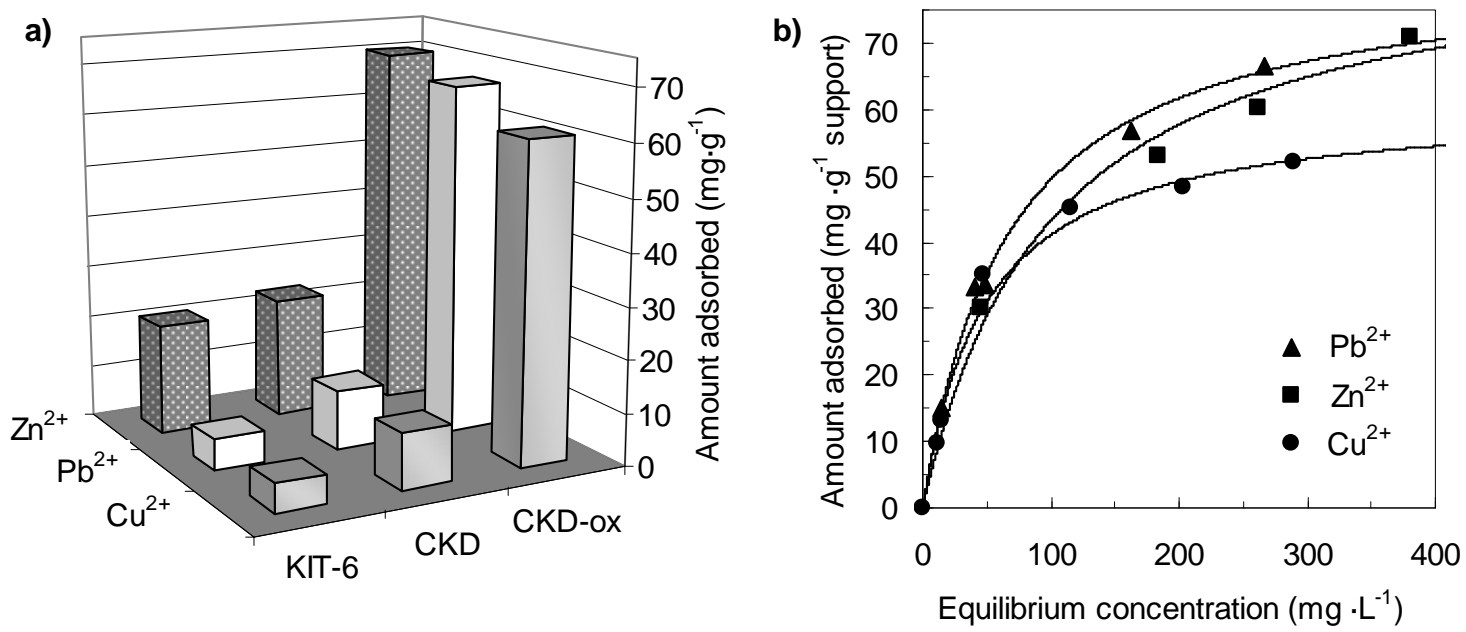

Figure 7. 


\section{References}

[1] F. Hoffmann, M. Cornelius, J. Morell, M. Fröba, Angew. Chem. Int. Ed., 45 (2006) 3216-3251.

[2] G.J.D. Soler-illia, C. Sanchez, B. Lebeau, J. Patarin, Chem. Rev., 102 (2002) 40934138.

[3] C.H. Huang, K.P. Chang, H.D. Ou, Y.C. Chiang, C.F. Wang, Microp. Mesop. Mater., 141 (2011) 102-109.

[4] W. Shaobin, Microp. Mesop. Mater., 117 (2009) 1-9.

[5] J. Lei, J. Fan, C.Z. Yu, L.Y. Zhang, S.Y. Jiang, B. Tu, D.Y. Zhao, Microp. Mesop. Mater., 73 (2004) 121-128.

[6] F. Schuth, W. Schmidt, Adv. Mat., 14 (2002) 629-638.

[7] P. Srinivasu, V.V. Balasubramanian, L. Kumaresan, D.P. Sawant, X. Jin, S. Alam, K. Ariga, T. Mori, A. Vinu, J. Nanosci. Nanotechnol., 7 (2007) 3250-3256.

[8] Z. Yan, S. Tao, J. Yin, G. Li, Journal of Materials Chemistry, 16 (2006) 2347-2353.

[9] M.C. Bruzzoniti, A. Prelle, C. Sarzanini, B. Onida, S. Fiorilli, E. Garrone, J. Sep. Sci., 30 (2007) 2414-2420.

[10] G.-P. Hao, W.-C. Li, D. Qian, G.-H. Wang, W.-P. Zhang, T. Zhang, A.-Q. Wang, F. Schüth, H.-J. Bongard, A.-H. Lu, J. Am. Chem. Soc., 133 (2011) 11378-11388.

[11] Y. Zhang, F.L.-Y. Lam, X. Hu, Z. Yan, Chem. Commun., (2008) 5131-5133.

[12] P. Valle-Vigon, M. Sevilla, A.B. Fuertes, Microp. Mesop. Mater., 134 (2010) 165174.

[13] P. Valle-Vigón, M. Sevilla, A.B. Fuertes, Appl. Surf. Sci. 261 (2012) 574-583.

[14] P.A. Bazula, A.H. Lu, J.-J. Nitz, F. Schüth, Microp. Mesop. Mater., 108 (2008) 266-275.

[15] A. Vinu, K.Z. Hossian, P. Srinivasu, M. Miyahara, S. Anandan, N. Gokulakrishnan, T. Mori, K. Ariga, V.V. Balasubramanian, J. Mater. Chem., 17 (2007) 1819-1825.

[16] A. Stein, Z. Wang, M.A. Fierke, Adv. Mater., 21 (2009) 265-293.

[17] C. Moreno-Castilla, M.A. Ferro-Garcia, J.P. Joly, I. Bautista-Toledo, F. CarrascoMarjn, J. Rivera-Utrilla, Langmuir, 11 (1995) 4386-4392.

[18] D. Zhao, Q. Huo, J. Feng, B.F. Chmelka, G.D. Stucky, J. Am.Chem. Soc., 120 (1998) 6024-6036.

[19] F. Kleitz, S. Hei Choi, R. Ryoo, Chem. Commun., (2003) 2136-2137.

[20] H. Nishihara, Y. Fukura, K. Inde, K. Tsuji, M. Takeuchi, T. Kyotani, Carbon, 46 (2008) 48-53.

[21] C. Moreno-Castilla, F. Carrasco-Marín, A. Mueden, Carbon, 35 (1997) 1619-1626.

[22] M. Kruk, M. Jaroniec, A. Sayari, Langmuir, 13 (1997) 6267-6273.

[23] H.P. Boehm, Carbon, 32 (1994) 759-769.

[24] M. Seredych, J. Lison, U. Jans, T.J. Bandosz, Carbon, 47 (2009) 2491-2500.

[25] G. Socrates, Infrared and Raman Characteristic Group Frequencies Wiley New York 2005.

[26] R. Schlögl, Carbons, in: Preparation of Solid Catalysts, Wiley-VCH Verlag GmbH, 2008, pp. 150-240.

[27] J.-H. Zhou, Z.-J. Sui, J. Zhu, P. Li, D. Chen, Y.-C. Dai, W.-K. Yuan, Carbon, 45 (2007) 785-796.

[28] E. Desimoni, G.I. Casella, A. Morone, A.M. Salvi, Surf. Interface Anal., 15 (1990) 627-634.

[29] J.L. Figueiredo, M.F.R. Pereira, Catal. Today, 150 (2010) 2-7. 
[30] Y. Otake, R.G. Jenkins, Carbon, 31 (1993) 109-121.

[31] N. Li, X. Ma, Q. Zha, K. Kim, Y. Chen, C. Song, Carbon, 49 (2011) 5002-5013.

[32] J.L. Figueiredo, M.F.R. Pereira, M.M.A. Freitas, J.J.M. Órfão, Carbon, 37 (1999) 1379-1389.

[33] F.A. Batzias, D.K. Sidiras, J. Hazard. Mater., 141 (2007) 668-679.

[34] K.Y. Ho, G. McKay, K.L. Yeung, Langmuir, 19 (2003) 3019-3024.

[35] Y. Dong, B. Lu, S. Zang, J. Zhao, X. Wang, Q. Cai, J. Chem. Technol. Biotechnol., 86 (2011) 616-619.

[36] D. Zhang, Y. Ma, H. Feng, H. Luo, J. Chen, Y. Hao, Korean J. Chem. Eng., 29 (2012) 775-780.

[37] A. Derylo-Marczewska, A.W. Marczewski, S. Winter, D. Sternik, Appl. Surf. Sci., 256 (2010) 5164-5170.

[38] M. Rafatullah, O. Sulaiman, R. Hashim, A. Ahmad, J. Hazard. Mater., 177 (2010) 70-80.

[39] M. Machida, B. Fotoohi, Y. Amamo, L. Mercier, Appl. Surf. Sci., 258 (2012) 7389-7394.

[40] A. Shahbazi, H. Younesi, A. Badiei, Chem. Eng. J., 168 (2011) 505-518.

[41] T.M. Abdel-Fattah, S.M.S. Haggag, M.E. Mahmoud, Chem. Eng. J., 175 (2011) 117-123. 


\section{Supporting Information}

for

\section{Carboxyl-functionalized mesoporous silica-carbon composites}

\section{as highly efficient adsorbents in liquid phase}

Patricia Valle-Vigón, Marta Sevilla and Antonio B. Fuertes*

Instituto Nacional del Carbón (CSIC), P. O. Box 73, 33080-Oviedo, Spain

* Corresponding author (E-mail: abefu@incar.csic.es)

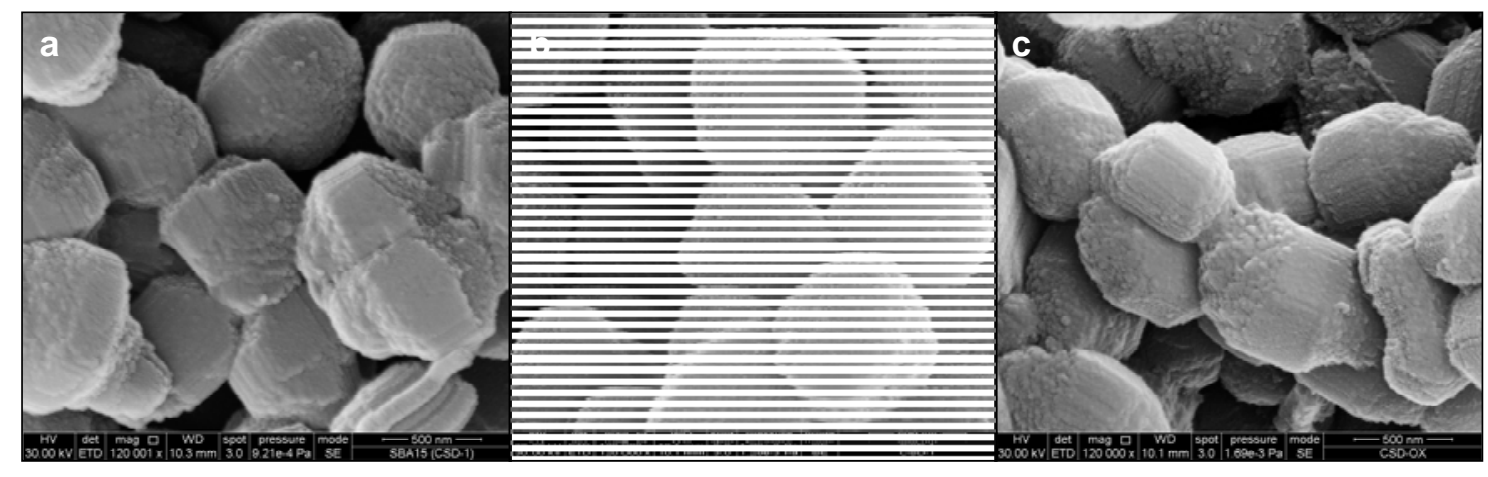

Figure S1. SEM micrographs of (a) SBA-15, (b) CSD and (c) CSD-ox. 

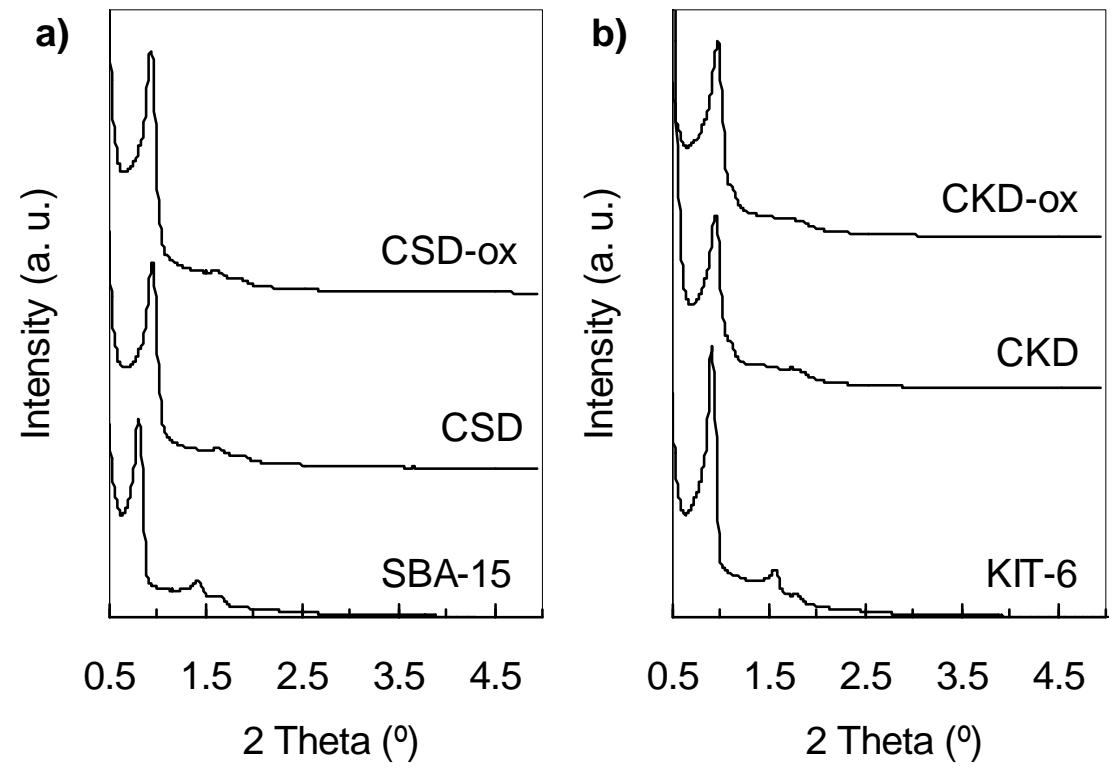

Figure S2. XRD patterns in the low-angle region of the mesostructured silica materials and the corresponding silica-carbon composites before and after the oxidation step.
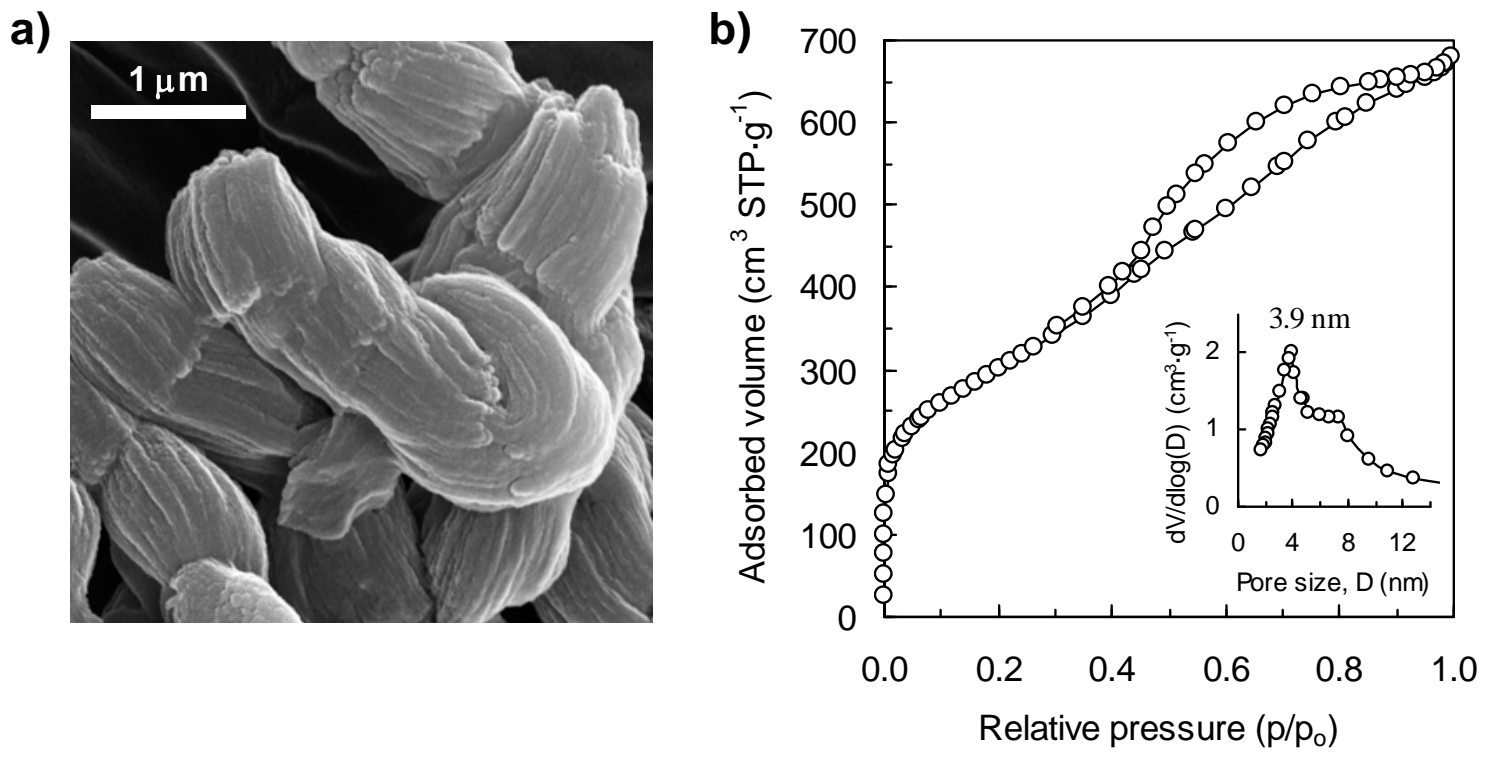

Figure S3. (a) SEM image and (b) sorption isotherm and pore size distribution (inset) corresponding to the templated carbon obtained by dissolving the silica skeleton of a SBA-15/carbon composite. 
